\title{
Nitric oxide content and apoptosis rate in steroid-induced avascular necrosis of the femoral head
}

\author{
RUI BAI ${ }^{1}$, WANLIN LIU ${ }^{2}$, AIQING ZHAO ${ }^{3}$, ZHENGQUN ZHAO $^{2}$ and DIANMING JIANG ${ }^{4}$ \\ ${ }^{1}$ Graduate School of Chongqing Medical University, Chongqing; ${ }^{2}$ Department of Pediatric Orthopedics, \\ Second Affiliated Hospital of Inner Mongolia Medical University, Hohhot, Inner Mongolia; \\ ${ }^{3}$ Department of Computed Tomography, Affiliated Hospital of Inner Mongolia Medical University, \\ Hohhot, Inner Mongolia; ${ }^{4}$ Department of Orthopedics, First Affiliated Hospital of \\ Chongqing Medical University, Chongqing, P.R. China
}

Received April 26, 2014; Accepted March 31, 2015

DOI: $10.3892 /$ etm.2015.2521

\begin{abstract}
The aim of the present study was to explore the effect on nitric oxide (NO) content and osteocyte apoptosis of steroid-induced avascular necrosis of the femoral head (SANFH) in an animal model of SANFH. A total of 40 Japanese white rabbits, 5 months of age and weighing $2.5 \pm 0.5 \mathrm{~kg}$, were randomly divided into groups A (hormone + endotoxin group), B (endotoxin + normal saline group), C (normal saline + hormone group) and D (control group). Following the establishment of the model, a blood sample was taken from the heart of each animal and centrifuged; the levels of NO in the serum were detected. The bilateral femoral heads were conventionally dissected, fixed, decalcified and stained with hematoxylin and eosin. Subsequently, the empty bone lacunae were counted under an optical microscope. Changes in osteocyte morphology were observed using electron microscopy and osteocyte apoptosis was detected with a terminal deoxynucleotidyl transferase dUTP nick end labeling assay. The percentage of empty bone lacunae in group A was significantly higher compared with that in groups $\mathrm{B}, \mathrm{C}$ and $\mathrm{D}(\mathrm{P}<0.01)$; however, there was no significant difference in percentage among groups B, C and D. The NO content in group A was significantly higher compared with that in groups $\mathrm{B}, \mathrm{C}$ and D $(\mathrm{P}<0.01)$; however, there was no significant difference in NO content among groups B, C and D. The osteocyte apoptosis index in group A was significantly higher compared with that in the other groups $(\mathrm{P}<0.01)$; there was no significant difference among groups B, C and D. NO content was positively correlated with osteocyte apoptosis index ( $\mathrm{r}=0.707)$. Thus, the present study found that NO content and the osteocyte apoptosis index were increased in SANFH, and that they play an
\end{abstract}

Correspondence to: Dr Dianming Jiang, Department of Orthopaedics, First Affiliated Hospital of Chongqing Medical University, 1 Friendship Road, Chongqing 400016, P.R. China

E-mail: dianmingjiang@126.com

Key words: hormone, osteonecrosis, apoptosis, nitric oxide important role in SANFH. The content of NO was positively correlated with the osteocyte apoptosis index, indicating that NO induces apoptosis.

\section{Introduction}

Previous clinical studies have found that the primary cause of femoral head necrosis is steroid hormones, which is the main reason for the disease (1). The occurrence of femoral head necrosis is associated with the administration method and dose of the hormones, and is affected by individual differences and sensitivities (2). It is not possible to make an early diagnosis for the majority of patients with certainty, and effective procedures for prevention and treatment are lacking as the pathogenesis remains unknown. During the previous decade, the apoptosis theory has received increased research attention along with the development of molecular biology; apoptosis of osteocytes and osteoblasts is considered to play an important role in steroid-induced avascular necrosis of the femoral head (SANFH) (3). SANFH and the apoptosis of osteocytes and osteoblasts are considered to be closely associated (4). Chan and Mok (5) found that the excessive use of hormones influenced the formation mechanisms of osteoblasts and osteoclasts in bone marrow, and decreased the rate of skeleton formation and bone mineral density (BMD), in addition to inducing the apoptosis of mature osteoblasts and osteoclasts. The authors hypothesized that the primary effects of the hormones included inducing the apoptosis of mature osteoblasts and osteoclasts, inhibiting osteoclastogenesis and reducing the bone turnover rate, leading to the development of apoptosis and resulting in femoral head necrosis. The majority of the target molecules of caspase-3, which regulates the differentiation of numerous cells, including skeletal muscle cells, neurocytes and lymphocytes, have been identified. The target molecules include certain cytoskeletal proteins, cyclins, kinases and transcription regulators (6). Previous studies have demonstrated that the expression of caspase- 3 is upregulated, along with an increase in apoptosis rate, in various types of cells, and the association between caspase- 3 and apoptosis can also be observed in osteocytes, osteoblasts and chondrocytes (7-9). Therefore, cell apoptosis and the apoptotic pathway 
in SANFH are the primary focus of studies concerning the pathogenesis of the disease.

In the present study, a rabbit model of SANFH was established and blood samples were collected for centrifugation to detect changes in nitric oxide (NO) content in serum; the osteoblast apoptosis index was also detected in the bilateral femoral head. The aim of the study was to investigate the association between SANFH and NO content variation, as well as the association between this variation and osteocyte apoptosis.

\section{Materials and methods}

Experimental animal. A total of 40 healthy adult Japanese white rabbits (male and female), 5 months old and weighing $2.5 \pm 0.5 \mathrm{~kg}$, were provided by the Beijing Fuhao Animal Breeding Center (Beijing, China) with a certificate issued by the National Institute of Medical Experiments (no. 20090132). The rabbits were fed with a standard diet and housed individually in cages. The Ethics Committee of The First Affiliated Hospital of Chongqing Medical University (Chongqing, China) approved of this study.

Experimental instruments. A $\mathrm{H}-700 \mathrm{H}$ electron microscope (Hitachi, Tokyo, Japan), LKB 2800 ultramicrotome (LKB, Bromma, Sweden), T22N visible spectrophotometer (Shanghai Qinghua Scientific Education Equipment Co., Ltd., Shanghai, China), paraffin embedding machine (EG1150 C; Leica Biosystems Co. Ltd., Shanghai, China), an ultra-thin slicing machine (Sweden $\mathrm{L}_{\mathrm{KB}} 2800$; Leica Biosystems), JD-801 medical image analysis system (Jiangsu Xunjie Co. Ltd., Jiangsu, China), Millipore RU water purification system (EMD Millipore, Billerica, MA, USA), and a CX41 microscope (Olympus Corporation, Tokyo, Japan) were used in the study. The above instruments were provided by the Molecular Biology Research Center and Electron Microscopy Center of Inner Mongolia Medical University (Hohhot, China).

Reagents. Escherichia coli (E. coli) endotoxin (provided by the National Institute for the Control of Pharmaceutical and Biological Products, Beijing, China), methylprednisolone (Pharmacia \& Upjohn Co., Brussels, Belgium), NO detection kit using a nitrate reductase assay (Beijing 4A Biotech Co., Ltd., Beijing, China) and the terminal deoxynucleotidyl transferase dUTP nick end labeling (TUNEL) assay apoptosis detection kit (Wuhan Boster Company, Wuhan, China) were used. Other conventional reagents, including hematoxylin and eosin (H\&E) staining and ethylenediaminetetraacetic acid (EDTA) decalcification solutions, were provided by the Molecular Biology Research Center of the Inner Mongolia Medical University.

Grouping and establishment of the experimental animal model. Experimental animals were divided into 4 groups by a random number table method. Group $\mathrm{A}$, with 10 rabbits, was the model group; rabbits were intravenously injected with the $E$. coli endotoxin, twice at $24 \mathrm{~h}$ intervals with $100 \mu \mathrm{g} / \mathrm{kg}$ each time. Following the second injection of the E. coli endotoxin, the rabbits were intramuscularly injected with methylprednisolone, 3 times at $24 \mathrm{~h}$ intervals with $20 \mathrm{mg} / \mathrm{kg}$ each time. Group B, with 10 rabbits, was the endotoxin group; rabbits were intravenously injected with $E$. coli endotoxin, twice at $24 \mathrm{~h}$ intervals with $100 \mu \mathrm{g} / \mathrm{kg}$ each time. Following the second injection of $E$. coli endotoxin, rabbits were intramuscularly injected with normal saline, 3 times at $24 \mathrm{~h}$ intervals with $20 \mathrm{mg} / \mathrm{kg}$ each time. Group C, with 10 rabbits, was the hormone group; rabbits were intravenously injected with normal saline, twice at $24 \mathrm{~h}$ intervals with $100 \mu \mathrm{g} / \mathrm{kg}$ each time. Following the second injection of normal saline, the rabbits were intramuscularly injected with methylprednisolone, 3 times at $24 \mathrm{~h}$ intervals with $20 \mathrm{mg} / \mathrm{kg}$ each time. Group D, with 10 rabbits, was the control group; rabbits were intravenously injected with normal saline, twice at $24 \mathrm{~h}$ intervals with $100 \mu \mathrm{g} / \mathrm{kg}$ each time. Following the second injection of normal saline, the rabbits were intramuscularly injected with normal saline, 3 times at $24 \mathrm{~h}$ intervals with $20 \mathrm{mg} / \mathrm{kg}$ each time. Four weeks after the final injection, a $5 \mathrm{ml}$ blood sample was taken from the heart of each animal and the animals were subsequently sacrificed by air embolism. The bilateral femoral head samples were collected under sterile conditions (Fig. 1A).

Specimen preparation. Blood samples were centrifuged for $10 \mathrm{~min}$ at $1,989 \mathrm{x} \mathrm{g}$ at $20^{\circ} \mathrm{C}$ and the upper serum was stored at $-20^{\circ} \mathrm{C}$ for testing. One femoral head was fixed in $5 \%$ glutaraldehyde solution and the other in $10 \%$ formaldehyde solution, for one week. Bone tissue was decalcified for 40 days using 15\% EDTA and embedded in paraffin (Fig. 1B).

Histomorphological observation under an optical microscope. The paraffin-embedded tissue blocks were cut into slices with a thickness of $4 \mu \mathrm{m}$ and stained with H\&E. The histopathological changes were observed under an optical microscope at x100 and x400 magnifications. The histopathological changes were classified according to the Matsui method (10) as follows: Grade 0, intact trabecular bone and bone marrow without pathological changes; grade 1, simple bone marrow necrosis without trabecular bone necrosis, manifested as cell lysis, nuclear fragmentation, karyolysis in bone marrow cells and hypochromatosis, blurred nuclear boundaries as well as fused boundaries in fat cells; and grade 2, trabecular necrosis and bone marrow necrosis; in addition to the pathological changes of grade 1, there are also empty lacunae accompanied by newly formed adherent bone. The degree of pathological change associated with bone cell necrosis was indicated by the percentage of empty bone lacunae. At x100 magnification, 5 fields were randomly selected to determine the empty bone lacunae and calculate the percentage of empty bone lacunae. The ratio of empty lacunae in the subchondral femoral region has been widely accepted as a histological indicator of SANFH. The normal proportion of empty lacunae in the subchondral femoral region of an adult rabbit is $8-12 \%$ (11). Therefore, with the same sample preparation procedure, an empty lacunae ratio in the subchondral femoral region greater than that number indicates the presence of osteonecrosis.

Observation under a transmission electron microscope. The femoral heads were fixed with $5 \%$ glutaraldehyde and decalcified. The bone mass $0.2-0.3 \mathrm{~cm}$ under the femoral head cartilage was cut into a $1-\mathrm{mm}^{3}$ section, decalcified with $5 \%$ nitric acid for 2-3 h, fixed in $1 \%$ osmium tetroxide for $1 \mathrm{~h}$, dehydrated in graded alcohol solutions and embedded using 
epoxy resin (Epon 812; Beijing Dalike Technology Co. Ltd., Beijing, China). The anhydrous ethanol was gradually replaced by mixing the embedding medium with anhydrous ethanol at a ratio of 1:1,2:1 and 3:1. Subsequently, the sample was saturated with embedding medium by incubation in a $37^{\circ} \mathrm{C}$ thermostat incubator (DC-0506; FDL Technology Information Co. Ltd., Nanjing, China) for $24 \mathrm{~h}$, aggregated at $60^{\circ} \mathrm{C}$ in an oven for $48 \mathrm{~h}$, cut into 50-nm ultrathin slices using an ultramicrotome and stained (double staining with uranyl acetate and lead citrate). The ultrathin slices were observed under the $\mathrm{H}-700 \mathrm{H}$ electron microscope.

Determination of NO content in the rabbit serum by a nitrate reductase assay. NO is chemically active and reacts with molecular oxygen to generate $\mathrm{NO}_{2}$. This transforms into $\mathrm{NO}_{3}{ }^{-}$and $\mathrm{NO}_{2}^{-}$, and $95 \% \mathrm{NO}_{2}^{-}$transforms into $\mathrm{NO}_{3}^{-}$within $1 \mathrm{~h}$. Thus, the determination of the serum level of $\mathrm{NO}_{2}^{-}$is inaccurate for determining the level of NO. However, the sum of serum $\mathrm{NO}_{2}^{-}$and $\mathrm{NO}_{3}^{-}$levels is an accurate indicator of $\mathrm{NO}$ level. Thus, in the present assay, $\mathrm{NO}_{3}^{-}$was specifically reduced to $\mathrm{NO}_{2}^{-}$by nitrate reductase and the NO content was indicated by color intensity; that is, the optical density (OD) value indicated the NO level. The reagents used for the test were as follows: Reagent 1, $6.0 \mathrm{ml}$ phosphate-buffered saline (PBS); reagent 2, $6.0 \mathrm{ml}$ nitrate reductase; reagent 3 , $30 \mathrm{ml}$; reagent 4, $30 \mathrm{ml}$; reagent $5,1 \mathrm{ml} 100 \mu \mathrm{mol} / \mathrm{ml}$ potassium nitrate solution. The use of the reagents in the process is detailed in Table I. The mixed solution was maintained at room temperature for $10 \mathrm{~min}$. A $0.5-\mathrm{cm}$ cuvette was used; the blank solution was set as the zero point and the OD values of the measuring and standard solutions were calculated. The OD values were calculated as follows: Nitric oxide $(\mu \mathrm{mol} / \mathrm{l})=$ measuring solution OD/standard solution OD $\times 100$.

Detection of osteoblast apoptosis by TUNEL assay. The assay was performed according to kit instructions (TUNEL Apoptosis Assay kit; Wuhan Boster Company). The prepared paraffinized tissue slices were placed in a dyeing tank, washed twice with dimethyl benzene and anhydrous ethanol and once with 95 and $75 \%$ ethanol, respectively. The slices were subsquently washed for 5 min with PBS. Proteinase K solution $(20 \mu \mathrm{g} / \mathrm{ml})$ was added and the slices were incubated for $15 \mathrm{~min}$ at room temperature. The hydrolyzed tissue proteins were removed and the slices were washed 4 times with distilled water. PBS containing $2 \%$ hydrogen peroxide was added, left for $5 \mathrm{~min}$ and the slices were washed twice with PBS. Residual liquid was removed using filter paper and 2 drops terminal deoxynucleotidyl transferase (TdT) were immediately added to each slice. The residual liquid was again removed using filter paper and $54 \mu 1 \mathrm{TdT}$ enzyme buffer was then titrated onto each slice for $1 \mathrm{~h}$. Washing and reaction terminating solutions preheated to $37^{\circ} \mathrm{C}$ were added and the slices were maintained at $37^{\circ} \mathrm{C}$ for $30 \mathrm{~min}$. The slices were washed 3 times with PBS, and 2 drops peroxidase-labeled anti-digoxin antibody (1:16) were added for $30 \mathrm{~min}$. After washing 4 times with PBS, freshly prepared $0.05 \%$ 3,3'-diaminobenzidine was added to each slice, left for $5 \mathrm{~min}$ and the slices were washed 4 times using distilled water. The slices were counterstained with methyl green at room temperature for $10 \mathrm{~min}$. They
Table I. Determination of nitric oxide (NO) content in rabbit serum by the nitrate reductase method.

\begin{tabular}{lccc}
\hline Material & $\begin{array}{c}\text { Measuring } \\
\text { tube }(\mathrm{ml})\end{array}$ & $\begin{array}{c}\text { Standard } \\
\text { tube }(\mathrm{ml})\end{array}$ & $\begin{array}{c}\text { Blank } \\
\text { tube }(\mathrm{ml})\end{array}$ \\
\hline Sample & 0.1 & - & - \\
Potassium nitrate & - & 0.1 & - \\
Double distilled water & - & - & 0.1 \\
Reagent 1 & 0.1 & 0.1 & 0.1 \\
Reagent 2 & 0.1 & 0.1 & 0.1 \\
Reagent 3 & 0.5 & 0.5 & 0.5 \\
Reagent 4 & 0.5 & 0.5 & 0.5 \\
\hline
\end{tabular}

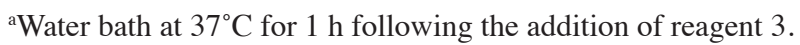
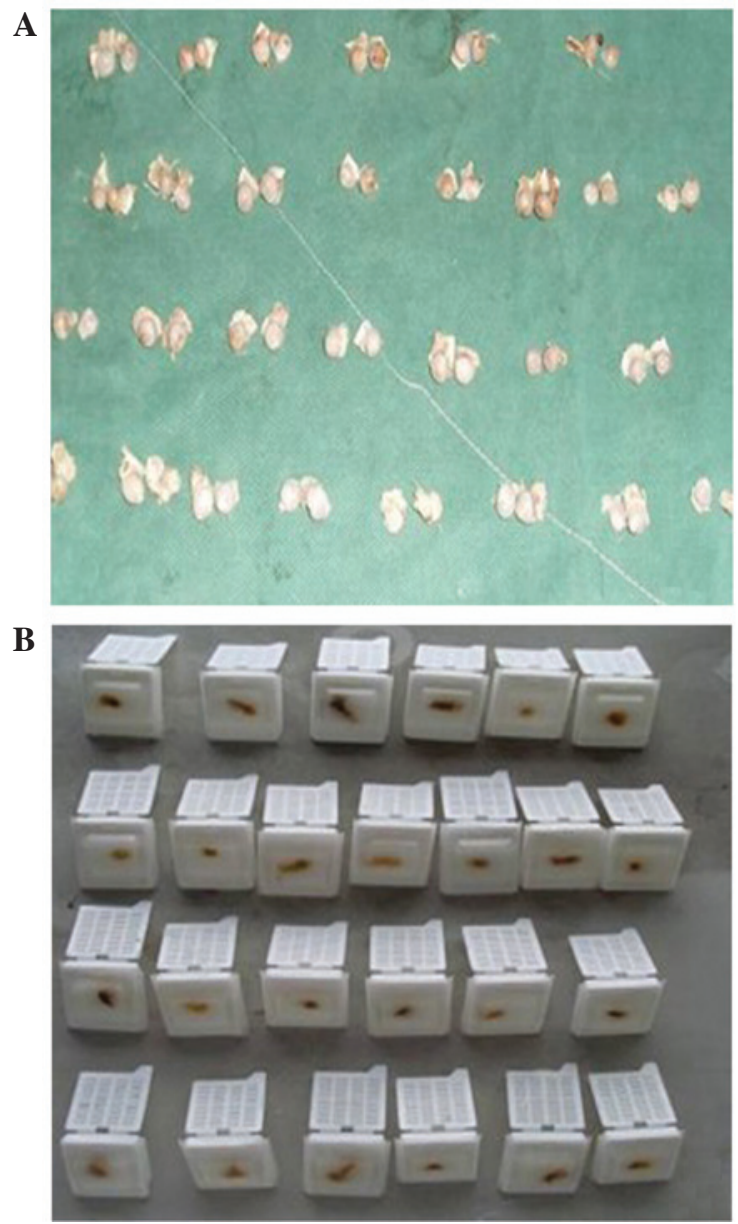

Figure 1. (A) Collected bilateral femoral head samples and (B) the samples embedded in paraffin.

were subsequently washed 3 times with distilled water and then 3 times with $100 \%$ butanol. The slices were dehydrated 3 times using dimethylbenzene, mounted, dried and observed under an optical microscope. TUNEL-positive staining, which appeared tan or brown, was identified in the nucleus with a few positive granules located in the cytoplasm. Five visual fields were randomly selected at high-magnification for each slice, 50 osteocytes were counted in each field and the apoptotic 

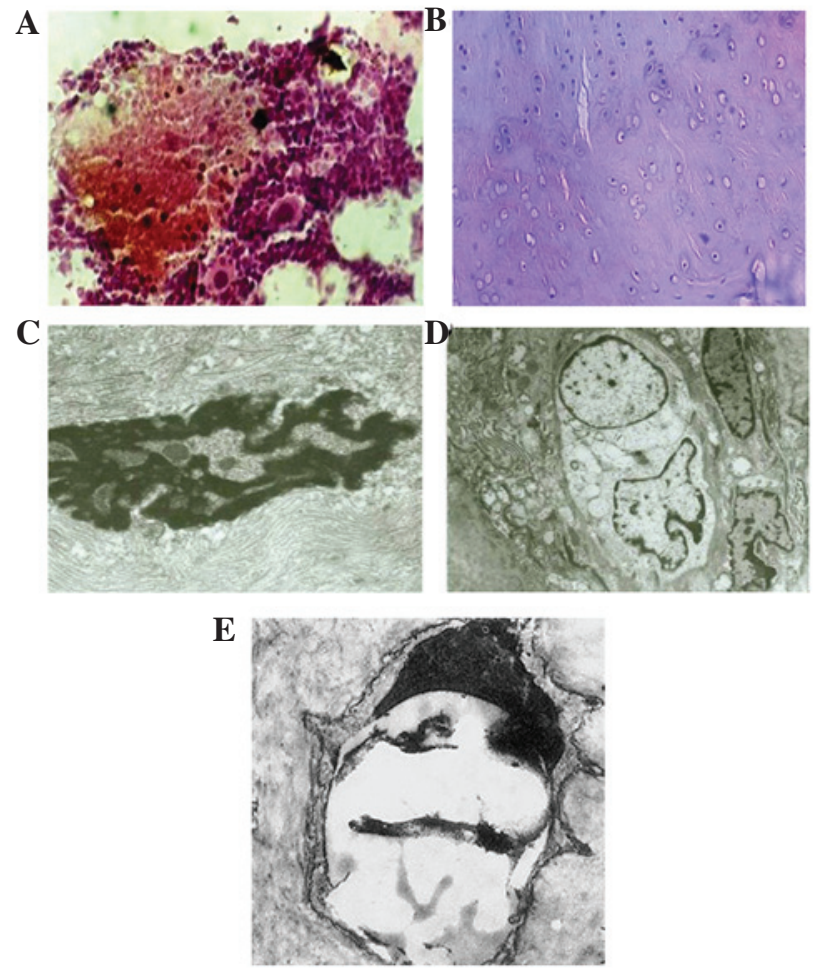

Figure 2. Bone structure of the rabbits in the hormone and endotoxin group. (A) The intramedullary structure was disordered with bone marrow hemorrhagic necrosis (H\&E staining; magnification, x6,000). (B) Bone cell necrosis resulted in empty lacunae (H\&E staining; magnification, $\mathrm{x} 100$ ). (C) Bone cell disrupted by necrosis. The nuclear membrane disappeared, chromatin was irregular and there were no organelles present (magnification, $\mathrm{x} 10,000$ ). (D) Bone cell necrosis was demonstrated by large vacuoles in the cytoplasm. (E) Apoptotic bone cell (TUNEL staining; magnification, $\mathrm{x} 10,000)$. H\&E, hematoxylin and eosin; TUNEL, terminal deoxynucleotidyl transferase dUTO nick end labeling assay.

index was calculated (apoptotic cell number/total cell number in the fields).

Statistical analysis. All data are presented as mean \pm standard deviation. Data were statistically analyzed by analysis of variance (ANOVA) using SPSS 13.0 software (SPSS, Inc., Chicago, IL, USA). Pairwise comparisons of multiple samples were carried out with Fisher's least significant difference test. The linear correlation between NO content and the apoptosis ratio in group A was analyzed. $-1 \leq \mathrm{r} \leq+1$ indicated that the two variables linearly correlated with one other; positive and negative values of $r$ indicate a positive and negative correlation, respectively. $\mathrm{P}<0.05$ was considered to indicate a statistically significant difference.

\section{Results}

Histomorphological observation. No significant difference in the general morphology of the femoral heads in the 4 groups was identified by observation with the naked eye. One femoral head was partially separated from the femoral neck in group A.

Changes observed under the optical microscope. Typical osteonecrosis was observed in the femoral head slices of
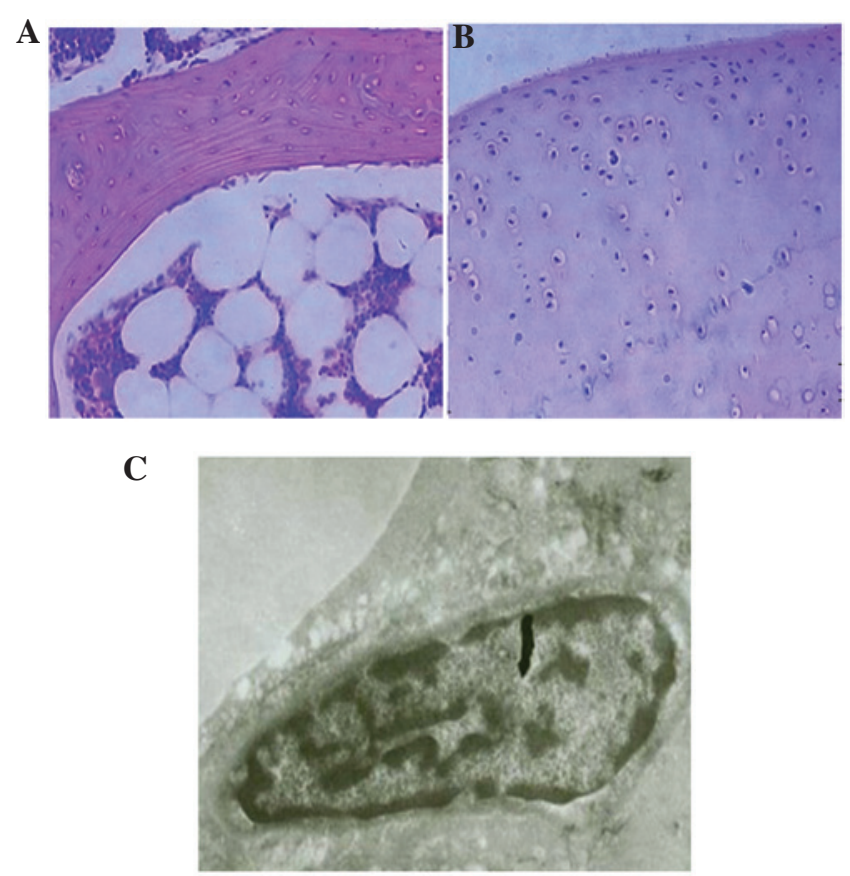

Figure 3. Bone structure of a rabbit from the control group. (A) The femoral intramedullary structure was clear (H\&E staining; magnification, x6,000). (B) Lacuna in the bone cells could be clearly observed (H\&E staining; magnification, $x 100$ ). (C) The cell membrane was uniform, and rough endoplasmic reticulum could be observed in the intracytoplasm. Nuclear chromatin was uniformly distributed and the stromal collagen fibers were in alignment (magnification, x10,000). H\&E, hematoxylin and eosin.

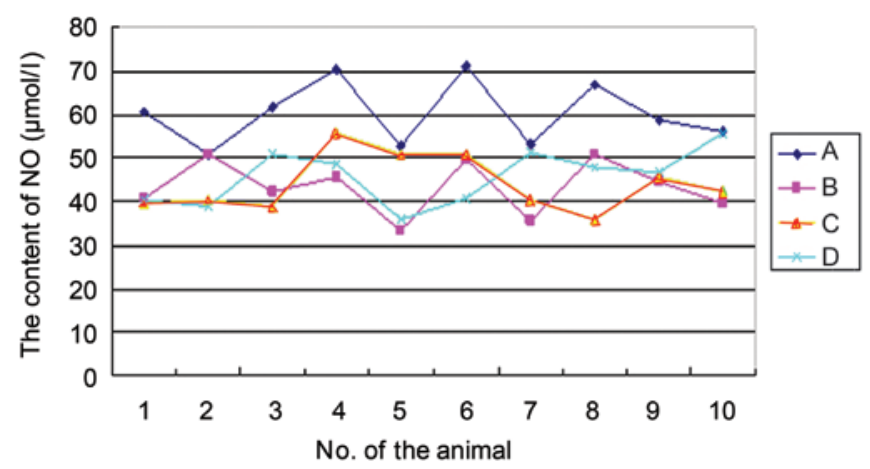

Figure 4. Nitric oxide (NO) content in the animals of each group

group A; it was mainly located under the cartilage. Bone trabeculae became scattered and thin, or even fractured, with structural disorder and fragmentation. The number of hematopoietic cells was reduced. Furthermore, the osteoblasts were arranged irregularly and the number of empty bone lacunae increased significantly. The volume of fat cells in the bone marrow increased, with certain fat cells becoming fused into vacuoles (Fig. 2A and B). In the femoral head slices in group B, the bone trabeculae in the femoral head biopsy were intact, regularly arranged and compacted. Bone cells with centrally located large nuclei were visible and there were numerous bone marrow hematopoietic cells. There were relatively few fat cells with normal morphology. In the group $\mathrm{C}$ slices, there was no visible osteonecrosis; bone trabeculae became scattered and thin, with mild structural disorders. There was an increase in bone marrow cell 
Table II. Classification of the histopathological changes in the bone samples by the Matsui method.

No. of the experimental animal

\begin{tabular}{lllllllllll}
\cline { 2 - 9 } Group & 1 & 2 & 3 & 4 & 5 & 6 & 7 & 8 & 9 & 10 \\
\hline A & 2 & 1 & 2 & 2 & 1 & 1 & 1 & 0 & 2 & 1 \\
B & 0 & 1 & 0 & 0 & 0 & 0 & 0 & 0 & 0 & 0 \\
C & 1 & 0 & 0 & 1 & 0 & 0 & 1 & 0 & 0 & 0 \\
D & 0 & 0 & 0 & 0 & 0 & 0 & 0 & 0 & 0 & 0 \\
\hline
\end{tabular}

Table III. Nitric oxide (NO) content and osteocyte apoptosis index in the animals of group A.

\begin{tabular}{lcccccccccc}
\hline & \multicolumn{10}{c}{ No. of the experimental animal } \\
\cline { 2 - 12 } Variable & 1 & 2 & 3 & 4 & 5 & 6 & 7 & 8 & 9 & 10 \\
\hline NO content $(\mu \mathrm{mol} / \mathrm{l})$ & 60.57 & 50.68 & 61.72 & 70.34 & 52.77 & 70.90 & 53.24 & 66.75 & 58.77 & 56.25 \\
Apoptotic index $(\%)$ & 27 & 29 & 30 & 35 & 32 & 34 & 26 & 33 & 30 & 30 \\
\hline
\end{tabular}

lysis and the number and volume of bone marrow fat cells. In the femoral head slices of group $\mathrm{D}$, the bone trabeculae were intact, regularly arranged and compacted. Bone cells with centrally located large nuclei were visible; there were numerous bone marrow hematopoietic cells and relatively normal fat cells (Fig. 3A and B). The classification of the histopathological changes in the bone samples by the Matsui method is detailed in Table II.

The empty bone lacuna rate in group A was significantly higher compared with that in groups $\mathrm{B}, \mathrm{C}$ and $\mathrm{D}(\mathrm{P}<0.01)$; there was no significant difference in the rate among groups $\mathrm{B}, \mathrm{C}$ and D. There were numerous empty bone lacunae in group $\mathrm{A}$ and the empty bone lacunae rate was $29.10 \pm 3.67 \%$. In groups $\mathrm{B}, \mathrm{C}$ and $\mathrm{D}$, the osteocytes in the rabbit femoral bone marrow were stained homogeneously with normal morphology and were moderately distributed; the empty bone lacunae rates in these three groups were 9.10 \pm 2.60 , $9.50 \pm 2.01$ and $8.01 \pm 2.36 \%$, respectively $(\mathrm{F}=137.88 ; \mathrm{P}<0.001)$.

Changes observed under the electron microscope. In the femoral head slices in group A, the osteocytes were disintegrated and necrotic, the nuclei were fragmented and the nuclear membrane was discontinuous or had completely disappeared. The majority of the organelles had partially or completely disappeared. Apoptotic bodies could be observed and there were large numbers of lysosomes and secretory granules in the cytoplasm. Bleeding was observed within the bone marrow (Fig. 2C and D).

The ultrastructure of osteocytes and bone marrow cells were normal in groups B and D (Fig. 3C).

Changes in NO content. The NO content in group A was significantly higher compared with that in groups $\mathrm{B}, \mathrm{C}$ and $\mathrm{D}(\mathrm{P}<0.01)$; there was no significant difference in $\mathrm{NO}$ content among groups B, C and D. The NO content was $60.19 \pm 7.26,43.25 \pm 6.11$,
44.04 \pm 6.44 and $45.75 \pm 6.47 \mu \mathrm{mol} / 1$ in groups A, B, C and D, respectively (Fig. 4; F=14.73; $\mathrm{P}<0.001$ ).

Osteocyte apoptosis observed with TUNEL staining. TUNEL-positive staining, which appeared tan or brown, was located in the nucleus with a few positive granules located in the cytoplasm. Five randomly-selected visual fields were observed at high-magnification for each slice and 50 osteocytes in each field were counted to calculate the apoptotic index. There were few apoptotic osteocytes in the bone samples from groups B, $\mathrm{C}$ and D. However, there were positive cells with visible brown particles in the nuclei of the bone samples from group A and numerous apoptotic osteocytes in the bone marrow tissue (Fig. 3E). The osteocyte apoptosis index in group A was significantly higher compared with that in groups $\mathrm{B}, \mathrm{C}$ and $\mathrm{D}(\mathrm{P}<0.01)$; there was no significant difference in the osteocyte apoptosis index among groups B, C and D. The osteocyte apoptosis indices were $30.80 \pm 5.14,6.90 \pm 1.92,6.60 \pm 1.27$ and $6.10 \pm 1.95$ in groups A, B, C and D, respectively $(\mathrm{F}=14.73 ; \mathrm{P}=<0.001)$.

Linear association between NO content and osteocyte apoptosis index. The apoptosis index in group A was $\mathrm{r}=0.707$ which meant that the NO content and osteocyte apoptosis index positively correlated with one other. The NO content and osteocyte apoptosis index in each animal of group A are detailed in Table III.

\section{Discussion}

SANFH, which is induced by the long-term or extensive short-term use of adrenocorticotropic hormone, has received much attention in the medical field. The number of clinical cases has been increasing since it was first reported by Pietrograndi in 1957 (12). SANFH, which is mainly bilateral and involves extensive necrosis, frequently occurs in young 
and middle-aged individuals. This disease is more serious than idiopathic avascular necrosis of the femoral head, with a higher disability rate (13). A large number of cases have emerged since 'atypical pneumonia' in 2010 (14). Investigating the etiology of SANFH remains a research hotspot in the field (15).

Steroid hormones have been widely accepted as an important cause of SANFH and numerous theories have been proposed concerning the pathogenesis, including fat embolism, high intraosseous pressure, enlarged fat cells, lipid droplet aggregation in osteocytes, osteoporosis and cumulative osteocyte dysfunction (16). However, the exact mechanism remains incompletely understood. Furthermore, the above theories do not clearly explain the occurrence of osteocyte necrosis as no cell swelling and inflammation accompany the characteristic necrosis of SANFH. Steroidal hormones, such as glucocorticoids, have been demonstrated to induce apoptosis (17). They reduce the number of osteocytes by promoting the apoptosis of osteoblasts and osteocytes, and they also affect the function of osteoblasts, retard osteogenesis and induce cell apoptosis, which results in bone loss. The present study further demonstrated the role of cell apoptosis in SANFH.

NO, an important bioactive substance, plays an important role in the regulation of normal body function and disease occurrence. Its major physiological functions include dilating vascular smooth muscle, and inhibiting the aggregation of platelets, platelet and leukocyte adhesion and hyperplasia of the vascular smooth muscle $(18,19)$. Hormones can damage vascular endothelial cells in which NO is produced and inhibit the activity of NO synthase (NOS). The appropriate concentration of NO maintains normal vasomotor center function to achieve the effective perfusion of organs. Vascular spasms, hyperplasia of the vascular wall, stenosis, platelet aggregation, leukocyte adhesion and thrombosis occur if NO content decreases. Avascular necrosis of femoral head is induced if these pathological changes occur in the femoral head and peripheral vasculature. NO is considered to be an important regulator of osteocyte apoptosis. A certain amount of NO has been revealed to bond with superoxide anions to reduce the level of oxygen free radicals and prevent their injuring effects on the microvasculature and synovium (20). Another study found that the content of inducible and endothelial NOS in osteoblasts, osteoclasts and bone marrow cells increased significantly in patients with osteonecrosis, while it was extremely low in patients with osteoarthritis (21). It has been suggested that NO may damage bone tissue in SANFH through the following mechanisms (22). Firstly, a high concentration of NO inhibits a variety of enzymes associated with the mitochondrial electron transport system and the citric acid cycle, including citrate synthase and aconitase. NO associates with the iron-sulfur centers of these enzymes to reduce the cellular iron level and thus inhibit the mitochondrial electron transport chain to cause cellular damage. Secondly, NO reacts with the superoxide anion $\mathrm{O}_{2}{ }^{--}$and generates the peroxynitrite anion $\mathrm{ONOO}^{-}$, which is relatively stable under alkaline conditions; however, under acidic conditions it rapidly decomposes into the free radicals $\mathrm{OH}^{\bullet}$ and $\mathrm{NO}_{2}{ }^{\circ}$ with strong toxicity. These two free radicals have very strong oxidizing activity and cytotoxicity, resulting in bone damage. NO has a dual function in bone absorption and inhibits the growth of osteoblasts or even has toxic effects during bone formation (1). However, the effect of NO on the process of SANFH development is unclear. The opinions of researchers concerning how NO content changes during SANFH are not consistent. Certain studies have shown that that the content increases while others have demonstrated the opposite (21). The dispute may be due to the different time-points at which the levels are detected. In the present study, NO content increased during the early period (week 4) of the SANFH model, which corresponded with osteocyte apoptosis.

Lee et al (23) studied the bone specimens of patients with nontraumatic osteonecrosis by immunohistochemistry and found that the NOS content was increased. The authors hypothesized that hormones or alcohol had direct cytotoxic effects on osteocytes, resulting in an increase in the NO content that mediated apoptosis. Thus, they concluded that osteocyte apoptosis played an important role in the pathogenesis of femoral head osteonecrosis. The results of the present study indicated that NO is associated with cell apoptosis and acts as an important apoptotic modulator; the exact mechanism for this remains to be further investigated.

In conclusion, in the present study, the precoagulation status was simulated by intravenously injecting $E$. coli endotoxin into rabbits and applying high-dose hormone to establish animal models of SANFH. Morphological and ultrastructural characteristics of the femoral head were observed and a comprehensive analysis was carried out by an immunohistochemical method. The results showed that NO content and the osteocyte apoptosis index were increased during the SANFH process, and indicated that increases in NO content and the osteocyte apoptosis index play important roles in SANFH. Furthermore, NO content was found to be positively correlated with the osteocyte apoptosis index, indicating that NO is able to induce cell apoptosis. Future studies are required to investigate the exact pathway for this and whether NO has a dual function.

\section{References}

1. Chen S, Li J, Peng H, Zhou J and Fang H: Administration of erythropoietin exerts protective effects against glucocorticoid-induced osteonecrosis of the femoral head in rats. Int J Mol Med 33: 840-848, 2014.

2. Saito M,Ueshima K,Fujioka M, et al: Corticosteroid administration within 2 weeks after renal transplantation affects the incidence of femoral head osteonecrosis. Acta Orthop 85: 266-270, 2014.

3. Nishida K, Yamamoto T, Motomura G, Jingushi S and Iwamoto Y: Pitavastatin may reduce risk of steroid-induced osteonecrosis in rabbits: A preliminary histological study. Clin Orthop Relat Res 466: 1054-1058, 2008.

4. Takano-Murakami R, Tokunaga K, Kondo N, Ito T, et al: Glucocoriticoid inhibits bone regeneration after osteonecrosis of the femoral head in aged female rats. Tohoku J Exp Med 217: 51-58, 2009.

5. Chan KL and Mok CC: Glucocorticoid-induced avascular bone necrosis: Diagnosis and management. Open Orthop J 6: 449-457, 2012.

6. Chen C, Yang S, Feng Y, et al: Impairment of two types of circulating endothelial progenitor cells in patients with glucocorticoid-induced avascular osteonecrosis of the femoral head. Joint Bone Spine 80: 70-76, 2013

7. Tan G, Kang PD and Pei FX: Glucocorticoids affect the metabolism of bone marrow stromal cells and lead to osteonecrosis of the femoral head: A review. Chin Med J (Engl) 125: 134-139, 2012.

8. Zou W, Yang S, Zhang T, et al: Hypoxia enhances glucocorticoid-induced apoptosis and cell cycle arrest via the PI3K/Akt signaling pathway in osteoblastic cells. J Bone Miner Metab Sep 18,2014 (Epub ahead of print). 
9. Ye J, Wu G, Li X, et al: Millimeter wave treatment inhibits apoptosis of chondrocytes via regulation dynamic equilibrium of intracellular free $\mathrm{Ca}^{2+}$. Evid Based Complement Alternat Med 2015: 464161, 2015

10. Ohzono K, Takaoka K, Saito S, Saito M, Matsui M and Ono K: Intraosseous arterial architecture in nontraumatic avascular necrosis of the femoral head. Microangiographic and histologic study. Clin Orthop Relat Res 277: 79-88, 1992.

11. Eberhardt AW, Yeager-Jones A and Blair HC: Regional trabecular bone matrix degeneration and osteocyte death in femora of glucocorticoid-treated rabbits. Endocrinology 142: 1333-1340, 2001.

12. Pietrograndi V and Mastromarino R: Osteopatia da prolungata trattamento cortisonico. Ortop Traum Appar Mar 25: 791-810, 1957 (In Italian)

13. Fukushima W, Fujioka M, Kubo T, et al: Nationwide epidemiologic survey of idiopathic osteonecrosis of the femoral head. Clin Orthop Relat Res 468: 2715-2724, 2010.

14. Qiu NH and Zhang W: Infectious atypical pneumonia after the etiology and treatment of femoral head necrosis. Chinese J Tissue Eng Res 17: 5525-5530, 2013.

15. Weinstein RS: Glucocorticoid-induced osteonecrosis. Endocrine 41: 183-190, 2012.

16. Kerachian MA, Cournoyer D, Harvey EJ, et al: New insights into the pathogenesis of glucocorticoid-induced avascular necrosis: Microarray analysis of gene expression in a rat model. Arthritis Res Ther 12: R124, 2010.
17. Kameda H, Amano K, Nagasawa $\mathrm{H}$, et al: Notable difference between the development of vertebral fracture and osteonecrosis of the femoral head in patients treated with high-dose glucocorticoids for systemic rheumatic diseases. Intern Med 48: 1931-1938, 2009.

18. Chen RM, Liu HC, Lin YL, Jean WC, Chen JS and Wang JH: Nitric oxide induces osteoblast apoptosis through the de novo synthesis of Bax protein. J Orthop Res 20: 295-302, 2002.

19. Buerk DG, Barbee KA and Jaron D: Nitric oxide signaling in the microcirculation. Crit Rev Biomed Eng 39: 397-433, 2011

20. Bao F, Wu P, Xiao N, Qiu F and Zeng QP: Nitric oxide-driven hypoxia initiates synovial angiogenesis, hyperplasia and inflammatory lesions in mice. PLoS One 7: e34494, 2012.

21. Calder JD, Buttery L, Revell PA, et al: Apoptosis - a significant cause of bone cell death in osteonecrosis of femoral head. J Bone Joint Surg Br 86: 1209-1213, 2004.

22. Hu M, Zhao H, Dong X, Luo D and Zhou X: General and light microscope observation on histological changes of femoral heads between SANFH rabbit animal models and it were intervened by Osteoking Zhongguo Zhong Yao Za Zhi 35: 2912-2916, 2010 (In Chinese)

23. Lee JS, Lee JS, Roh HL, Kim CH, Jung JS and Suh KT: Alterations in the differentiation ability of mesenchymal stem cells in patients with nontraumatic osteonecrosis of the femoral head: Comparative analysis according to the risk factor. J Orthop Res 24: 604-609, 2006. 